\title{
Estado nutricional e a estimativa de consumo dos nutrientes em mulheres do programa UCS sênior
}

\author{
Nutricional status and the estimate consumption of nutrients in women participating \\ in the UCS senior program
}

\author{
Sandra Czarnobai Benincáa, Josiane Sivierob ${ }^{b}$ Carin Weirich Gallonc \\ a Acadêmica do Curso de Graduação em Nutrição da Universidade de Caxias do Sul (UCS). \\ 'Nutricionista. Doutora em Ciências da Saúde pela Pontifícia Universidade Católica do Rio Grande do Sul (PUCRS). Docente da UCS. \\ c Nutricionista. Mestre em Ciências Médicas pela Universidade Federal do Rio Grande do Sul (UFRGS). Docente da UCS.
}

Objetivo: Avaliar o estado nutricional e o consumo dos macro e micronutrientes em mulheres de um programa de extensão.

Materiais e Métodos: Estudo transversal que foi realizado com mulheres $\geq 50$ anos. As variáveis analisadas foram: sociodemográficas, antropométricas e consumo alimentar. Correlacionou-se o estado nutricional com o consumo alimentar.

Resultados: A partir das 176 mulheres, verificou-se que a média de idade foi 63,2 anos $( \pm 7,4)$. A predominância do nível de escolaridade foi médio completo ou mais $(58,5 \%)$, excesso de peso $(52,3 \%)$ e risco muito aumentado para Doenças Cardio Vasculares (DCV) pela Circunferência Abdominal (CA) em 78,4\%. A média do consumo de macronutrientes ficou em $57,7 \%$ para carboidrato, 17,2\% para proteína e 25\% para lipídeos. Na correlação entre Índice de Massa Corporal (IMC) com CA, dados demográficos e consumo alimentar, houve associação positiva significativa entre IMC e CA $(p<0,001)$. Também houve associação negativa significativa entre IMC com nível de escolaridade $(p=0,041)$, calorias do café da manhã $(p=0,040)$ e fibras $(p=0,008)$. Já na análise de correlação entre CA com dados demográficos e consumo alimentar, houve associação positiva significativa entre CA com idade $(p=0,039)$ e proteínas, $(p=0,011)$. Também houve associação negativa significativa entre CA com nível de escolaridade $(p=0,005)$ e fibras $(p=0,007)$.

Conclusão: Encontrou-se uma elevada prevalência de excesso de peso e a CA como fator de risco para DCV. Correlações entre o estado nutricional e o consumo alimentar também foram demonstradas. A educação nutricional é fundamental para que as mulheres possam adequar a sua alimentação e, consequentemente, melhorar seu estado nutricional.

Palavras-chave: consumo alimentar; estado nutricional; índice de massa corporal; circunferência abdominal.

\section{ABSTRACT}

Objective: To evaluate the nutritional status and the consumption of macro and micronutrients in women participating in an extension program.

Materials and Methods: Cross-sectional study including women older than 50 years. The variables analyzed were socio demographic, anthropometric and food consumption, correlating the nutritional status with the food consumption.

Results: From 176 women, the average age found was 63.2 years old ( \pm 7.4$)$. The predominant scholarship level was high school completed or higher (58.5\%), overweight (52.3\%) and high risk for cardio vascular disease (CVD) due to the Abdominal Circumference (AC) in $78.4 \%$. The consumption of macronutrients was $57.7 \%$ carbohydrates, $17.2 \%$ protein and $25 \%$ lipids. When analyzing the correlation between Body Mass Index (BMI) and AC, a positive association $(p<0.001)$ was identified. There was also a negative association between BMI with the scholarship level $(p=0.041)$, with calories in the breakfast $(p=0.040)$ and fibers $(p=0.008)$. When analyzing the correlation between AC with demographic data and food consumption, there was a positive association with age $(p=0.039)$ and proteins $(p=0.011)$. There was also a negative association between $A C$ with the level of scholarship $(p=0.005)$ and fibers $(p=0.007)$.

Conclusion: A high prevalence of overweight was found and the AC as a risk factor for CVD. Correlations between the nutritional status with the food consumption were also found. The nutritional education is fundamental so that women can readapt their food consumption and, consequently, improve their nutritional status.

Keywords: food consumption; nutritional status; body mass index; abdominal circumference. 


\section{INTRODUÇÃO}

O envelhecimento é um processo caracterizado por alterações fisiológicas, psicológicas e sociais que ocorrem no decorrer dos anos. Estas alterações afetam o estado nutricional que, por sua vez, se relacionam com a saúde. É um fenômeno marcado por mudanças biopsicossociais específicas de cada indivíduo no decorrer dos anos ${ }^{1,2,3}$. De acordo com Organização Mundial de Saúde (OMS), "são considerados idosos, nos países desenvolvidos, os indivíduos com idade igual ou superior a 65 anos, ao passo que, nos países em desenvolvimento, considera-se a idade igual ou superior a 60 anos".

A população idosa no Brasil está aumentando a cada ano e as projeções apontam que para 2025 haverá cerca de 32 milhões de pessoas com idade maior ou igual a 60 anos, sendo a maioria composta por mulheres. Com esse número, o país ocupará o sexto lugar quanto ao contingente de idosos no mundo 4 . É incontestável que o Brasil experimenta, nos últimos anos, uma rápida transição nutricional. Chama a atenção o marcante aumento na prevalência de obesidade, consolidando-se como o agravo nutricional mais importante, sendo associado a uma alta incidência de doenças crônicas não transmissíveis, como diabetes e doenças cardiovasculares ${ }^{5,6}$. Principalmente nos últimos 50 anos, são observadas alterações na qualidade e na quantidade da dieta e, associadas a mudanças no estilo de vida, nas condições econômicas, sociais e demográficas, observam-se repercussões negativas na saúde populacional nos países emergentes ${ }^{7}$. No Brasil, observa-se um declínio da desnutrição, porém aumento na prevalência de sobrepeso/ obesidade nas últimas décadas, apresentando como singularidade notável, uma carência nutricional (a anemia) e uma condição típica dos excessos alimentares: a obesidade ${ }^{7,8}$.

Segundo Pesquisa de Orçamento Familiar 20082009, o consumo alimentar no Brasil é, principalmente, constituído por alimentos de alto teor energético e baixo teor de nutrientes. Essas características configuram uma alimentação de risco para o desenvolvimento de obesidade e para DCNT. O consumo de alimentos in natura é cada vez menor e vem sendo substituído pelos processados e ultraprocessados ${ }^{9}$. Neste aspecto, em se tratando de hábitos alimentares é, portanto, a chamada dieta ocidental que tem sido apontada como geradora da transição nutricional ocorrida neste século, convergindo para uma dieta mais rica em ácidos graxos saturados, ácidos graxos trans, açúcares e alimentos refinados e reduzidos em carboidratos complexos e fibras. Essa alimentação aliada ao declínio progressivo da atividade física, produz alterações na composição corporal, principalmente o aumento de gordura ${ }^{10,11}$.
As mudanças na alimentação se devem à oferta crescente de alimentos industrializados, redução do tamanho da família, aumento da disponibilidade de alimentos e facilidade de acesso da população a alimentos muito calóricos, contendo altas concentrações de gorduras e açúcares ${ }^{12}$. O estilo de vida moderno e consequente irregularidade na rotina alimentar, como o fato de não fazer algumas refeições, em especial o desjejum, também pode ser uma das causas das alterações do estado nutricional. Existem algumas evidências de menor Índice Massa Corporal (IMC), circunferência da cintura, percentual de gordura e melhores níveis de glicose em jejum na população que faz regularmente café da manhã, hábito que vem se perdendo ao longo do tempo ${ }^{13}$.

Várias funções metabólicas e fisiológicas são alteradas com o passar dos anos as quais podem interferir na saúde e no estado nutricional ao longo da vida. O estado nutricional expressa o grau no qual as necessidades fisiológicas por nutrientes estão sendo alcançadas para manter a composição e as funções adequadas do organismo, resultantes do equilíbrio entre ingestão e necessidade de nutrientes. Assim, alterações do estado nutricional contribuem para o aumento da morbimortalidade devido a complicações relacionadas tanto à desnutrição como ao excesso de peso ${ }^{14}$.

A alimentação saudável e consequente manutenção do estado nutricional adequado são fatores essenciais para um envelhecimento bem sucedido ${ }^{1}$. Com base no exposto acima, este estudo teve como objetivo avaliar o estado nutricional e o consumo dos macro e micronutrientes em mulheres do programa de extensão UCS Sênior.

\section{MATERIAIS E MÉTODOS}

Trata-se de um estudo transversal. Os dados primários que foram analisados são provenientes do banco de dados do projeto NUTENV intitulado "Alimentação e Envelhecimento: estudo do perfil nutricional, antropométrico e a relação com fatores socioeconômicos e culturais de mulheres idosas participantes da Universidade da Terceira Idade da Universidade de Caxias do Sul (UCS)". A coleta das informações aconteceu no período de 2010-2015. No projeto foram incluídos os alunos matriculados no Programa de Extensão UCS Sênior da Universidade da Terceira Idade da UCS, com idade igual ou superior a 50 anos.

As entrevistas foram agendadas por amostra de conveniência mediante contato telefônico com os participantes, que demonstraram interesse em participar da pesquisa. Eles foram convidados a comparecer ao Laboratório de Avaliação Nutricional da UCS para aplicação do questionário e avaliação antropométrica por acadêmicas do curso de Nutrição, previamente treinadas, com duração de 30 a 60 minutos. Todos os participantes assinaram um 
termo de Consentimento Livre e Esclarecido (TCLE). Neste estudo, a amostra foi composta por 176 mulheres do banco de dados do projeto citado acima.

As variáveis demográficas selecionadas foram idade, escolaridade, estado civil e renda. As variáveis antropométricas foram peso $(\mathrm{kg})$ e altura $(\mathrm{m})$, índice de massa corporal $\left(\mathrm{IMC}=\mathrm{kg} / \mathrm{m}^{2}\right)$ e circunferência abdominal $(\mathrm{CA}=\mathrm{cm})$. Para obtenção do peso foi utilizada a balança de plataforma mecânica da marca Cauduro, $\mathrm{CH} 180^{\circledR}$, com divisões de $100 \mathrm{~g}$ e capacidades mínima e máxima de $2 \mathrm{~kg}$ e $180 \mathrm{~kg}$, respectivamente, na qual as mulheres foram pesadas com roupas leves e descalças. A altura foi verificada através do estadiômetro vertical acoplado na balança, no qual foram medidas na posição ortostática, com as mãos lateralizadas ao corpo e com a cabeça alinhada à linha do horizonte ${ }^{15}$.

O IMC foi calculado a partir da divisão do peso em quilos pelo quadrado da altura em metros, para as mulheres com idade entre 50 e 60 anos. A classificação utilizada foi segundo World Healt Organization (WHO) ${ }^{16}<18,5 \mathrm{~kg} / \mathrm{m}^{2}$ baixo peso, $>18,5 \mathrm{~kg} / \mathrm{m}^{2}$ e $<24,9 \mathrm{~kg} / \mathrm{m}^{2}$ eutrofia, $>25,0$ a $29,9 \mathrm{~kg} / \mathrm{m}^{2}$ sobrepeso e $>30 \mathrm{~kg} / \mathrm{m}^{2}$ obesidade. Para a classificação das mulheres idosas com idade superior a 60 anos foi utilizada a classificação segundo a Organização Panamericana de Saúde (OPAS) ${ }^{17}<23 \mathrm{~kg} / \mathrm{m}^{2}$ baixo peso, $>23 \mathrm{~kg} / \mathrm{m}^{2}$ a $<28 \mathrm{~kg} / \mathrm{m}^{2}$ eutrofia, $>28 \mathrm{~kg} / \mathrm{m}^{2}$ a $<30 \mathrm{~kg} / \mathrm{m}^{2}$ sobrepeso e $>30 \mathrm{~kg} / \mathrm{m}^{2}$ obesidade.

A CA foi mensurada com fita inelástica, ao redor do abdômen no nível da maior diâmetro, usualmente ao nível da cicatriz umbilical ${ }^{15}$ e classificada como: adequada para $<80 \mathrm{~cm}$, risco aumentado de $>80$ a $87 \mathrm{~cm}$ e risco muito aumentado $>88 \mathrm{~cm}$ segundo as definições das Diretrizes Brasileiras de Obesidade ${ }^{18}$.

O consumo alimentar foi avaliado a partir do recordatório de 24 horas (R24h) onde a participante respondeu detaIhadamente sobre todos os alimentos e bebidas ingeridos no dia anterior o modo de preparo e a marca comercial. Por meio do qual foi possível obter o consumo alimentar ingerido habitualmente, pois era questionado o quão habitual era o relato da ingestão alimentar relatado. O registro dos alimentos foi feito em medidas caseiras para posterior conversão destas medidas em gramas, utilizando-se como padrão de referência a Tabela de Pinheiro ${ }^{19}$, para posteriormente serem inseridas no software de nutrição Dietwin ${ }^{\circledR}$ Profissional 2008 para análise de cada nutriente. Os valores dos nutrientes foram comparados com as recomendações das Dietary References Intakes (DRIs) ${ }^{20}$ para mulheres $\geq 50$ anos, sendo que para os macronutrientes $45-65 \%$ para carboidratos (CHO), 10-35\% para proteínas (PTN) e 20-35\% para lipídios (LIP) foram considerados os parâmetros da Acceptable Macronutrient Distribution Ranges (AMDR) e para as vitaminas piridoxina
(B6) $1,3 \mathrm{mg} / \mathrm{dia}$, ácido fólico (B9) $320 \mu \mathrm{g} / \mathrm{dia}$, cianocobalamina (B12) $2 \mu \mathrm{g} /$ dia e os minerais: cálcio (Ca) $1000 \mathrm{mg} / \mathrm{dia}$, ferro (Fe) $5 \mathrm{mg} /$ dia e zinco (Zn) $6,8 \mathrm{mg} /$ dia foi a Estimated Average Requirement (EAR), para as fibras $21 \mathrm{~g} /$ dia utilizou se as Recommended Dietary Allowances Adequate Intake (RDA/Al). Foi avaliada a distribuição das calorias em relação às refeições e analisou-se o valor energético do café da manhã por meio da porcentagem das calorias totais.

As variáveis quantitativas foram descritas por média e desvio padrão ou mediana e amplitude interquartílica, dependendo da distribuição dos dados. A distribuição das variáveis foi avaliada pelo teste de Kolmogorv-Smirnov. As variáveis qualitativas foram descritas por frequências absolutas e relativas. Para avaliar a associação entre as variáveis, os testes da correlação linear de Pearson ou Spearman foram aplicadas. Conforme Callegari-Jacques ${ }^{21}$, coeficientes de correlação abaixo de 0,3 indicam associação de fraca intensidade, entre 0,3 e 0,59 de regular intensidade e iguais ou acima de 0,6 de forte intensidade. O nível de significância adotado foi de $5 \%(p<0,05)$ e as análises foram realizadas no programa Statistical Package for the Social Science (SPSS $\left.{ }^{\circledR}\right)$, versão 21.0.

Este estudo foi aprovado pelo Comitê de Ética em Pesquisa da Universidade de Caxias do Sul sob o protocolo no 061/2009. Respeitou os preceitos éticos contidos na Declaração de Helsinki (2008), bem como a legislação que regulamenta a ética em pesquisa no país, conforme resolução 466/2012.

\section{RESULTADOS}

A partir das 176 mulheres, verificou-se que a média de idade foi 63,2 anos $( \pm 7,4)$, com variação entre 51 e 86 anos. A predominância foi de mulheres casadas $(67,6 \%)$, com nível de escolaridade de médio completo ou mais (58,5\%), renda de três ou mais s.m. (61,9\%), excesso de peso $(52,3 \%)$ e risco muito aumentado para DCV pela CA (78,4\%). Essas características estão apresentadas na Tabela 1.

O perfil das mulheres, quanto ao consumo alimentar, está descrito na Tabela 2. O consumo de macronutrientes ficou distribuído, em média, com 57,7\% para $\mathrm{CHO}, 17,2 \%$ para PTN e $25 \%$ para lipídeos. O percentual médio do café da manhã em relação ao total de calorias foi de $22,1 \%( \pm 9,7)$. A média do consumo de fibras foi de $21,7 \mathrm{~g}( \pm 9,7)$.

A mediana do consumo de vitamina $B_{9}$ foi de $184 \mathrm{mcg}$ (Percentis 25-75:129-247). Em relação ao consumo de vitamina $B_{12}$, o consumo mediano foi de $2,27 \mathrm{mcg}$ (Percentis 25-75: 1,23-3,48). Quanto ao consumo de vitamina $B_{6}$, a mediana foi de 1,05 mcg (Percentis 25-75: 0,75-1,57). A mediana do consumo de cálcio foi de $629 \mathrm{mg}$ (Percentis 25-75: 445-858). O consumo mediano de zinco foi de 
6,9 mg (Percentis 25-75: 4,1-10,4). Por fim, a média do consumo de ferro foi de $10,7 \mathrm{mg}( \pm 4,1)$.

Ao realizar a correlação entre IMC com CA, dados demográficos e consumo alimentar, houve associação positiva significativa entre IMC com CA $(p<0,001)$, ou seja, quanto maior o IMC, maior a CA destas mulheres. Também houve associação negativa significativa entre IMC com nível de escolaridade $(p=0,041)$, calorias do café da manhã $(p=0,040)$ e consumo de fibras $(p=0,008)$. Quanto maior o nível de escolaridade, calorias do café da manhã e consumo

Tabela 1. Caracterização da amostra de mulheres participantes do programa de extensão UCS Sênior $(n=176)$.

\begin{tabular}{|c|c|}
\hline Variáveis & $n=176$ \\
\hline Idade (anos) - média $\pm D P$ & $63,2 \pm 7,4$ \\
\hline Sexo feminino $-\mathrm{n}(\%)$ & $176(100)$ \\
\hline \multicolumn{2}{|l|}{ Estado civil - $\mathrm{n}(\%)$} \\
\hline Solteira & $6(3,4)$ \\
\hline Casada & $119(67,6)$ \\
\hline Divorciada & $14(8,0)$ \\
\hline Viúva & $37(21,0)$ \\
\hline \multicolumn{2}{|l|}{ Nível de escolaridade - n (\%) } \\
\hline Fundamental incompleto & $41(23,3)$ \\
\hline Fundamental completo & $23(13,1)$ \\
\hline Médio incompleto & $9(5,1)$ \\
\hline Médio completo & $41(23,3)$ \\
\hline Superior incompleto & $13(7,4)$ \\
\hline Superior completo & $49(27,8)$ \\
\hline \multicolumn{2}{|l|}{ Renda - n (\%) } \\
\hline Até $1 \mathrm{~s} . \mathrm{m}$. & $16(9,1)$ \\
\hline De 1 a 2 s.m. & $23(13,1)$ \\
\hline De 2 a 3 s.m. & $28(15,9)$ \\
\hline De 3 a 4 s.m. & $34(19,3)$ \\
\hline Acima de $4 \mathrm{~s} . \mathrm{m}$. & $75(42,6)$ \\
\hline Peso (kg) - média $\pm D P$ & $70,4 \pm 13,5$ \\
\hline Altura $(\mathrm{m})-$ média $\pm \mathrm{DP}$ & $1,58 \pm 0,06$ \\
\hline IMC $\left(\mathrm{kg} / \mathrm{m}^{2}\right)-$ média $\pm \mathrm{DP}$ & $28,4 \pm 5,2$ \\
\hline \multicolumn{2}{|l|}{ Classificação do IMC - n (\%) } \\
\hline Baixo peso & $15(8,5)$ \\
\hline Eutrofia & $69(39,2)$ \\
\hline Sobrepeso & $38(21,6)$ \\
\hline Obesidade & $54(30,7)$ \\
\hline CA $(\mathrm{cm})$ - média $\pm \mathrm{DP}$ & $96,2 \pm 12,3$ \\
\hline \multicolumn{2}{|l|}{ Classificação da CA - n (\%) } \\
\hline Adequada & $13(7,4)$ \\
\hline Risco & $25(14,2)$ \\
\hline Risco muito aumentado & $138(78,4)$ \\
\hline
\end{tabular}

DP: desvio-padrão; n: número; \%: porcentagem; IMC: índice de massa corporal CA: circunferência abdominal; s.m.: salário mínimo. de fibras, menor o IMC. Apesar das associações significativas, é importante ressaltar que, exceto pela correlação com CA, as demais associações com IMC foram de fraca intensidade. Estas correlações estão descritas na Tabela 3.

Tabela 2. Consumo alimentar de uma amostra de mulheres participantes do programa de extensão UCS Sênior ( $n=176)$.

\begin{tabular}{|c|c|}
\hline Variáveis & $n=176$ \\
\hline Energia $(k c a l)-$ média $\pm D P$ & $1548 \pm 488$ \\
\hline Café da manhã (kcal) - média $\pm \mathrm{DP}$ & $332 \pm 158$ \\
\hline Café da manhã (\% kcal total) - média $\pm D P$ & $22,1 \pm 9,7$ \\
\hline $\mathrm{CHO}(\%)$ - média $\pm \mathrm{DP}$ & $57,7 \pm 9,1$ \\
\hline PTN (g) - média \pm DP & $66,0 \pm 23,6$ \\
\hline PTN (\%) - média \pm DP & $17,2 \pm 4,4$ \\
\hline 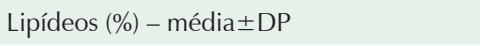 & $25,0 \pm 8,5$ \\
\hline Fibras (g) - média \pm DP & $21,7 \pm 9,7$ \\
\hline Vitamina $B_{9}(\mathrm{mcg})$ - md (P25-P75) & $184(129-247)$ \\
\hline Vitamina $B_{12}(\mathrm{mcg})-$ md (P25-P75) & $2,27(1,23-3,48)$ \\
\hline Vitamina $B_{6}(\mathrm{mcg})-$ md (P25-P75) & $1,05(0,75-1,57)$ \\
\hline Cálcio (mg) - md (P25-P75) & $629(445-858)$ \\
\hline Ferro $(\mathrm{mg})-$ média $\pm \mathrm{DP}$ & $10,7 \pm 4,1$ \\
\hline Zinco (mg) - md (P25-P75) & $6,9(4,1-10,4)$ \\
\hline
\end{tabular}

DP: desvio-padrão; n: número; \%: porcentagem; md: mediana; P25: percentil 25; P75: percentil75; CHO: Carboidrato; PTN: Proteína.

Tabela 3. Correlações entre IMC com CA, dados demográficos e consumo alimentar de uma amostra de mulheres participantes do programa de extensão UCS Sênior $(n=176)$.

\begin{tabular}{|c|c|c|}
\hline Variáveis & $\begin{array}{l}\text { Coeficiente de } \\
\text { correlação }\end{array}$ & $p$ \\
\hline Idade (anos) & $r=0,108$ & 0,154 \\
\hline Nível de escolaridade & $r_{s}=-0,154$ & $0,041^{*}$ \\
\hline Renda & $r_{s}=-0,091$ & 0,227 \\
\hline CA & $r=0,886$ & $<0,001^{*}$ \\
\hline Energia (kcal) & $r=-0,061$ & 0,420 \\
\hline Café da manhã (kcal) & $r=-0,155$ & $0,040^{*}$ \\
\hline Café da manhã (\% kcal total) & $r=-0,067$ & 0,379 \\
\hline $\mathrm{CHO}(\%)$ & $r=0,038$ & 0,613 \\
\hline PTN (g) & $r=-0,020$ & 0,794 \\
\hline PTN (\%) & $r=0,134$ & 0,076 \\
\hline Lipídeos (\%) & $r=-0,108$ & 0,154 \\
\hline Fibras (g) & $r=-0,198$ & $0,008^{*}$ \\
\hline Vitamina $B_{9}(\mathrm{mcg})$ & $r_{s}=0,005$ & 0,949 \\
\hline Vitamina $B_{12}(m c g)$ & $r_{s}=0,108$ & 0,156 \\
\hline Vitamina $B_{6}(\mathrm{mcg})$ & $r_{s}=0,062$ & 0,416 \\
\hline Cálcio (mg) & $r_{s}=-0,114$ & 0,131 \\
\hline Ferro (mg) & $r=-0,077$ & 0,308 \\
\hline Zinco (mg) & $r_{s}=0,084$ & 0,266 \\
\hline
\end{tabular}

$r_{s}$ : coeficiente de correlação de Spearman; r: coeficiente de correlação de Pearson.

* Associação estatisticamente significativa a 5\% de significância. 
$\mathrm{Na}$ análise de correlação entre CA com dados demográficos e consumo alimentar, houve associação positiva significativa entre CA com idade $(p=0,039)$ e consumo de proteínas em $\%(p=0,011)$, ou seja, quanto maior a idade e o consumo percentual de proteínas, maior a CA destas mulheres. Também houve associação negativa significativa entre CA com nível de escolaridade $(p=0,005)$ e consumo de fibras $(p=0,007)$. Quanto maior o nível de escolaridade e o consumo de fibras, menor a CA. Novamente, apesar das associações significativas, é importante ressaltar que as associações foram de fraca intensidade, conforme demonstra Tabela 4.

Tabela 4. Correlações entre CA com dados demográficos e consumo alimentar de uma amostra de mulheres participantes do programa de extensão UCS Sênio $(n=176)$.

\begin{tabular}{lcc}
\hline Variáveis & $\begin{array}{c}\text { Coeficiente de } \\
\text { correlação }\end{array}$ & $\boldsymbol{p}$ \\
\hline Idade (anos) & $\mathrm{r}=0,155$ & $0,039^{*}$ \\
Nível de escolaridade & $\mathrm{r}_{\mathrm{s}}=-0,212$ & $0,005^{*}$ \\
Renda & $\mathrm{r}_{\mathrm{s}}=-0,112$ & 0,140 \\
Energia (kcal) & $\mathrm{r}=-0,111$ & 0,144 \\
Café da manhã (kcal) & $\mathrm{r}=-0,143$ & 0,059 \\
Café da manhã $(\%$ kKcal total) & $\mathrm{r}=-0,062$ & 0,413 \\
CHO (\%) & $\mathrm{r}=-0,065$ & 0,392 \\
PTN (g) & $\mathrm{r}=-0,024$ & 0,748 \\
PTN (\%) & $\mathrm{r}=0,191$ & $0,011 *$ \\
Lipídeos $(\%)$ & $\mathrm{r}=-0,033$ & 0,663 \\
Fibras $(\mathrm{g})$ & $\mathrm{r}=-0,202$ & $0,007^{*}$ \\
Vitamina $\mathrm{B}_{9}(\mathrm{mcg})$ & $\mathrm{r}_{\mathrm{s}}=-0,033$ & 0,661 \\
Vitamina $\mathrm{B}_{12}(\mathrm{mcg})$ & $\mathrm{r}_{\mathrm{s}}=0,137$ & 0,071 \\
Vitamina $\mathrm{B}_{6}(\mathrm{mcg})$ & $\mathrm{r}_{\mathrm{s}}=0,048$ & 0,525 \\
Cálcio $(\mathrm{mg})$ & $\mathrm{r}_{\mathrm{s}}=-0,100$ & 0,185 \\
Ferro $(\mathrm{mg})$ & $\mathrm{r}=-0,076$ & 0,316 \\
Zinco $(\mathrm{mg})$ & $\mathrm{r}_{\mathrm{s}}=0,051$ & 0,504 \\
\hline
\end{tabular}

$r_{s}$ : coeficiente de correlação de Spearman; $r$ : coeficiente de correlação de Pearson; *associação estatisticamente significativa a 5\% de significância.

\section{DISCUSSÃO}

Neste estudo, destaca-se como principal achado que as mulheres que possuíam maior nível de escolaridade, que consumiam mais calorias no café da manhã e fibras, apresentavam menor Índice de Massa Corporal. Da mesma forma, quanto maior o nível de escolaridade e o consumo de fibras menor foi a Circunferência Abdominal. Cabe ressaltar, que apesar das associações significativas, estas foram de fraca intensidade. Na presente discussão os dados analisados estão descritos e foram relacionados com a literatura.
Em relação a expectativa de vida, esta tem aumentado entre as mulheres. Segundo o estudo de Cervato ${ }^{22}$, elas são mais participativas nas atividades socioculturais e estão mais sensibilizadas às necessidades demandadas para a promoção da saúde. Dados do IBGE ${ }^{23}$ demonstram que as mulheres são a maioria da população. As participantes deste estudo fazem parte do programa de extensão UCS Sênior Educação e Longevidade, que tem ampla adesão do público feminino ${ }^{24}$.

A compreensão do processo de envelhecimento, assim como a avaliação do estado nutricional e do consumo alimentar, se faz necessária entre as mulheres. Verificou-se, no presente estudo, que a maioria delas possuía um ótimo nível de escolaridade e renda. Esse fato pode proporcionar a oportunidade de buscarem e de se inserirem em atividades de lazer, integração e conhecimento ofertadas pela UCS Sênior.

Dentre os fatores que se avaliou no estado nutricional das mulheres, encontra-se o sobrepeso e a obesidade. Segundo a Pesquisa de Orçamento Familiar (POF) 2008-2009, a maior prevalência de sobrepeso e obesidade ocorre no sul do Brasil. A pesquisa também revela que o excesso de peso afeta $48 \%$ das mulheres e, destas, $16,9 \%$ são obesas. Conforme achados no presente estudo, obteve-se prevalência de 52,3\% de excesso de peso segundo a classificação do IMC, sendo que estudos realizados no Sul corroboram os resultados desta pesquisa. Conforme publicado por Kümpel ${ }^{25}$, encontrou-se uma prevalência de 49,6\% de sobrepeso/obesidade onde a maioria dos avaliados eram mulheres. Outro estudo realizado com 200 mulheres em $2012^{26}$ foi encontrado um IMC médio de $30,1 \pm 5,93 \mathrm{~kg} / \mathrm{m}^{2}$, resultado este semelhante ao encontrado no presente estudo $28,4 \pm 5,2 \mathrm{~kg} / \mathrm{m}^{2}$.

Com o passar dos anos, várias funções metabólicas e fisiológicas são alteradas e estas podem interferir na saúde e no estado nutricional ao longo da vida. A mulher, após os 50 anos, está propensa ao ganho ponderal de peso, assim como alterações da distribuição da gordura corporal, podendo estar relacionada à redução das necessidades energéticas, assim como a interrupção da função ovariana e a redução nos níveis de estrogênio, levando à diminuição da taxa metabólica basal. Ao associar as alterações metabólicas e fisiológicas com fatores extrínsecos, entre estes o comportamento alimentar inadequado, a mulher poderá ter um ganho ponderal de peso ao longo dos anos. Alguns estudos evidenciam que a frequência de excesso de peso não seria somente influenciada por fatores biológicos relacionados ao hipoestrogenismo, mas também por fatores psicossociais e pelo estilo de vida. O excesso de peso é um fator relevante no desenvolvimento de doenças crônicas não transmissíveis (DCNT) e vem sendo considerado um problema de saúde pública ${ }^{14,27,28,29}$. 
A média da CA encontrada foi de $96,2 \pm 12,3 \mathrm{~cm}$, sendo que $78,4 \%$ das mulheres possuíam um risco muito aumentado para o desenvolvimento de doenças cardiovasculares. Achados semelhantes em alguns estudos encontraram a mediana de $97 \mathrm{~cm}^{25}, 99,6 \mathrm{~cm}^{30}$ e $98,9 \mathrm{~cm}^{31} \mathrm{em}$ mulheres.

Durante o processo de envelhecimento, com a redistribuição progressiva da gordura, verifica-se um aumento de depósito de adiposidade. As mulheres passam a acumular mais gordura subcutânea, principalmente na região abdominal. Isso tem sido associado a alterações metabólicas nos níveis de glicose e lipídios que, por sua vez, relacionam-se com as doenças cardiovasculares, diabetes e hipertensão arterial sistêmica ${ }^{32}$.

Ao correlacionar o IMC e a CA houve associação positiva, ou seja, quanto maior o IMC, maior a CA. Com isso, maiores são os riscos para o desenvolvimento de DCV. Segundo Rezende $^{33}$, indivíduos com excesso de peso, principalmente com obesidade abdominal, estão mais expostos a fatores de risco cardiovasculares envolvidos na síndrome metabólica $\mathrm{e}$, consequentemente, a maior risco de morbidade e mortalidade quando não tratadas essas alterações. Quando avaliados, IMC e CA fornecem uma melhor associação da distribuição da gordura com as doenças crônicas ${ }^{34}$.

$\mathrm{Na}$ avaliação do consumo alimentar do presente estudo, utilizou-se o inquérito alimentar através do R24h, que é utilizado para qualificar e quantificar os alimentos consumidos diariamente por meio de relatos das 24 horas anteriores ${ }^{35}$. Estudos apontam que apenas um dia de R24h não representaria a ingestão habitual de um indivíduo devido à elevada variação no consumo de nutrientes ${ }^{36}$. Porém, coletar dados de vários dias de consumo alimentar se torna um procedimento que requer maior tempo de estudo e maior disponibilidade do avaliado ${ }^{37}$. Além disso, a omissão ao relatar o consumo de alguns alimentos, a dificuldade de memória em quantificar as porções e até mesmo a falta de compreensão das perguntas realizadas pelo entrevistador poderia promover um relato impreciso da ingestão energética ${ }^{38}$. Neste sentido, no presente estudo, visando minimizar a possível imprecisão do relato através do R24h, ao final do inquérito alimentar questionava-se se a ingestão relatada era habitual ou não.

Analisando o consumo alimentar das mulheres, verificou-se que a média do consumo de macronutrientes (57,7\% para $\mathrm{CHO}, 17,2 \%$ para PTN e 25\% para lipídios) ficou dentro dos parâmetros da AMDR. Dados do Inquérito de Alimentação da Pesquisa de Orçamento Familiar (POF) 2008-2009 da região Sul em mulheres também apontou o consumo de macronutrientes adequado, corroborando os dados de outros estudos realizados também na região $\mathrm{Su}^{39,40}$. O consumo adequado de macronutrientes é essencial. A queda na ingestão de nutrientes, secundária à anorexia do envelhecimento, é considerada um fator de risco importante no desenvolvimento e na progressão da sarcopenia ${ }^{41}$. O menor aporte de proteínas de alto valor biológico (PAVB) parece ser o problema mais significativo e pode ser alvo potencial de uma intervenção. $\mathrm{O}$ consumo de PAVB, ricas em aminoácidos essenciais, principalmente leucina, deve ser incentivado ${ }^{42}$. Já o aumento do consumo de lipídios e carboidratos, considerados pró-oxidantes, a elevada ingestão alimentar, especialmente de dietas hipercalóricas, e o aparecimento da obesidade têm sido associados ao aumento da produção de espécies reativas, induzindo o estresse oxidativo que pode ter importante papel nas alterações metabólicas ${ }^{43}$.

Ao verificar a ingestão das vitaminas, os valores medianos para $B_{9}$ e $B_{6}$ ficaram abaixo da recomendação, segundo a EAR. Ao correlacionar as vitaminas com o estado nutricional, não obtiveram associação estatisticamente significativa. É reconhecido que os micronutrientes possuem papel importante na prevenção de doenças prevalentes no Brasil. A ação antioxidante de algumas vitaminas pode reduzir a ocorrência de DCNT, pois têm função de proteção através das enzimas antioxidades endógenas e exógenas ${ }^{44}$.

Com o passar dos anos, os indivíduos são mais suscetíveis a carências nutricionais, sendo que a deficiência de vitamina $\mathrm{B}_{12}, \mathrm{~B}_{6}$ e $\mathrm{B}_{9}$, acomete frequentemente a população idosa. Esses déficits têm uma repercussão em parâmetros hematológicos, neurológicos, cardiovasculares e também no tecido ósseo, tornando o idoso mais predisposto a osteoporose e fraturas ósseas por fragilidade. Além disso, as deficiências dessas vitaminas também dificultam a execução de diversas reações enzimáticas ${ }^{45}$. Sabe-se que apenas algumas carências específicas recebem maior atenção e tem maior nível de evidência quanto à detecção e suplementação. A deficiência de cianocobalamina é mais comumente causada por má absorção da vitamina presente nos alimentos, geralmente devido à gastrite atrófica e a hipocloridria ${ }^{46}$.

Dentre os minerais, o consumo de cálcio apresentou inadequação entre as mulheres segundo a recomendação da EAR. O estudo de dados do Inquérito de Alimentação da Pesquisa de Orçamento Familiar (POF) 2008-2009 também mostrou uma inadequação do consumo de cálcio nas mulheres em todo o Brasil, corroborando outros estudos ${ }^{26,44}$ que encontraram valores semelhantes. Além disso, outra dificuldade em relação à adequação de cálcio deve-se ao fato de que a porcentagem de cálcio absorvido depende da quantidade total de cálcio elementar consumido de uma só vez. Quando a quantidade aumenta, a porcentagem de absorção diminui. A absorção é mais elevada em doses iguais ou inferiores a $500 \mathrm{mg}^{47}$. 
A ingestão adequada de cálcio é fundamental na manutenção da saúde óssea, na redução do risco de osteoporose e no controle da Pressão Arterial frequentes na faixa etária de adultos e idosos ${ }^{44}$. Além disso, o cálcio auxilia a regulação da termogênese e processo lipogênese/ lipólise. A ingestão de cálcio possivelmente se encontra relacionada ao controle do peso por diversos mecanismos. No trato intestinal, proporciona uma ligação com os ácidos graxos, limitando a sua absorção. A participação do mineral na regulação da temperatura corporal e no aumento da termogênese também tem sido apontada como efeito antiobesidade ${ }^{48}$.

Quando realizada a correlação entre IMC e consumo alimentar, o estudo encontrou associação negativa, estatisticamente significativa entre IMC e calorias do café da manhã, no presente estudo todas as mulheres realizavam o café da manhã. O hábito de tomar café da manhã frequentemente foi altamente associado com o consumo de alimentos apropriados e nutritivos, os quais têm uma repercussão positiva no peso corporal ${ }^{49}$. Também está associado com efeitos positivos na saúde, como aumento de saciedade, menor consumo energético diário, melhor controle da glicemia, menores riscos de obesidade e gordura visceral e menores riscos de comorbidades relacionadas ao peso: hipertensão, diabetes e doenças cardiovasculares ${ }^{50}$. O consumo adequado do café da manhã parece, também, auxiliar no controle de peso. Comparado aos lanches, a refeição matinal proporciona uma maior ingestão de vitaminas, minerais e fibras e menor ingestão de gorduras e colesterol $^{51}$. Um estudo que analisou os efeitos do hábito de tomar o café da manhã sobre alguns hormônios reguladores de apetite, comprovou que o grupo que consumia o café da manhã (CM) mais energético, em comparação a um jantar mais energético, apresentou maior perda de peso e redução da circunferência da cintura, da glicose em jejum, da insulina. A resistência à insulina (HOMA-IR) diminuiu significativamente no grupo $\mathrm{CM}$. Os níveis médios de triglicérides diminuíram 33,6\% no grupo CM, mas aumentaram $14,6 \%$ no grupo que fazia o jantar mais reforçado (J). Em resposta, a glicose diária total, insulina, grelina e as pontuações médias de fome foram significativamente menores, ao passo que as pontuações médias de saciedade foram significativamente maiores no grupo $\mathrm{CM}^{52}$.

Quanto ao consumo de fibras, encontrou-se dentro do recomendado pelas DRI's. Em sua correlação com o estado nutricional (IMC e CA) foi encontrada associação negativa, ou seja, quanto maior o consumo de fibras, menor é o IMC e CA. Estudo de Molz ${ }^{53}$, embora de forma não significativa, encontrou a mesma relação da presente pesquisa. Os efeitos positivos da fibra alimentar estão relacionados, em parte, ao fato de que uma parcela da fermentação de seus componentes ocorre no intestino grosso, o que produz impacto sobre a velocidade do trânsito intestinal, sobre o $\mathrm{pH}$ do cólon e sobre a produção de subprodutos com importante função fisiológica. Assim, o consumo adequado de fibras pode estar prevenindo várias doenças e também auxiliando na redução do peso corporal, bem como na redução dos níveis séricos do colesterol e no controle da glicemia. Alguns mecanismos estariam relacionados ao consumo adequado de fibras, como: aumento da saciedade, aumento do tempo de mastigação, menor consumo de calorias e redução da eficiência da absorção de outros alimentos no intestino delgado ${ }^{53,54}$. Também pode estar relacionado ao hábito de realizar o café da manhã.

Houve associação positiva significativa entre CA e consumo de proteínas, ou seja, quanto maior o consumo percentual de proteínas, maior a CA destas mulheres. Os alimentos ricos em proteína são geralmente ricos em lipídeos e esses, em excesso, são armazenados em depósitos subcutâneos, acarretando ganho de peso e aumento da gordura visceral5 ${ }^{55}$

Já na análise de correlação entre IMC, CA e dados demográficos, houve associação negativa significativa com nível de escolaridade e IMC e CA, ou seja, quanto maior o nível de escolaridade, menor o IMC e menor a CA. Estudo ${ }^{56}$ com obesos, sendo a maioria mulheres, encontrou a relação negativa entre IMC e escolaridade. Verificou-se, no presente estudo, que a maioria das mulheres possuía melhor nível de escolaridade e renda. Esse fato pode sugerir que estas tenham mais acesso a informações e serviços de saúde, modificando práticas alimentares e estilo de vida, o que pode estar contribuindo para um melhor estado nutricional e, consequentemente, uma melhor qualidade de vida.

Também se encontrou associação positiva entre CA e idade, ou seja, quanto maior a idade, maior a CA destas mulheres. O avançar da idade está associado a alterações na composição corporal, onde se observa uma redistribuição das mudanças sendo as principais a diminuição do músculo esquelético e o aumento do tecido adiposo. O aumento da gordura visceral ocorre, preferencialmente, na região abdominal. Há uma diminuição do tecido adiposo dos braços e pernas. Essas modificações acarretam ganho de peso e aumento dos riscos para o desenvolvimento de doenças crônicas não transmissíveis e da obesidade ${ }^{57,58}$.

Algumas limitações devem ser apontadas neste estudo. Ao realizar o inquérito alimentar R24h, este dependia da memória da entrevistada, o que pode ter influenciado nos valores energéticos e nutricionais relatados. Cabe ressaltar que a maioria das avaliadas possui um ótimo nível cognitivo, de esclarecimento, de escolaridade e de renda. Além disso, 
é importante destacar que as correlações não significam causalidade, já que sempre existe a possibilidade do relacionamento entre as variáveis ser influenciado por um terceiro fator, já que neste estudo não foi feita análise de regressão linear multivariada.

Os dados analisados permitem concluir que o presente estudo encontrou prevalência elevada de excesso de peso, assim como CA com risco muito aumentada para DCV, além de identificar algumas correlações entre o estado nutricional, consumo alimentar e dados sociodemográficos, no que diz respeito a IMC, CA, ao consumo de fibras, proteínas, calorias no café da manhã, à escolaridade e à idade. Importante salientar que mesmo que os macronutrientes e alguns micronutrientes encontravam-se dentro das recomendações, torna-se necessário reforçar as orientações sobre a composição nutricional dos alimentos, para que as mulheres possam adequar a sua alimentação e, consequentemente, melhorar seu estado nutricional.

\section{REFERÊNCIAS}

1. Freitas AMP, Philipi ST, Ribeiro SML. Listas de alimentos relacionadas ao consumo alimentar de um grupo de idosos: análises e perspectiva. Rev Bras Epidemiol. 2011;14(1):161-77. https://doi.org/10.1590/ S1415-790X2011000100015

2. Ferreira $A B, C$ Minanni $C A$, Paganini $C B L$, La Falce $T S$, Todeschini $A B$, Eras AE, Chekin G, Motta BR, Carneiro Júnior N. Programa de atenção particularizada ao idoso em unidades básicas de saúde. Saúde Soc. 2009;18(4):776-86. https://doi.org/10.1590/S010412902009000400020

3. Ferreira OGL, Maciel CS, Silva AO, Sá RCN, Silva P, Moreira MA. Significados atribuídos ao envelhecimento: idoso, velho e idoso ativo. Psico-USF. 2010;15(3):357-64. https://doi.org/10.1590/ S1413-82712010000300009

4. Instituto Brasileiro de Geografia e Estatística. Pesquisa de orçamentos familiares 2008-2009 [Internet]. [capturado 2016 Mar 16]. Disponível em: http://www.ibge.gov.br/home/xml/pof_2008_2009. shtm; 2010

5. Souza EB. Transição nutricional no Brasil: análise dos principais fatores. Cad UNIFOA. 2010;5(13):49-53.

6. Marinho CF, Beltrame MASB, Ferreira KK, Nobre JAS, Córdoba GMC. Consumo alimentar de usuários de uma Clínica-Escola de Nutrição do interior paulista. RASBRAN. 2017;8(1):52-7.

7. Batista Filho M, Rissin A. A transição nutricional no Brasil: tendências regionais e temporais. Cad Saúde Pública. 2003;19(1):S181-91. https://doi.org/10.1590/S0102-311X2003000700019

8. Batista Filho M, Souza Al, Miglioli TC, Santos MC. Anemia e obesidade: um paradoxo da transição nutricional brasileira. Cad Saúde Pública. 2008;24(2):S247-57. https://doi.org/10.1590/ S0102-311X2008001400010

9. Instituto Brasileiro de Geografia e Estatística. Pesquisa de orçamentos familiares 2008-2009: análise do consumo alimentar pessoal no Brasil. IBGE [Internet]. 2011 [capturado 2018 Out 02]. Disponível em: https://biblioteca.ibge.gov.br/visualizacao/livros/liv50063.pdf
10. Tardido AP, Falcão MC. O impacto da modernização na transição nutricional e obesidade. Rev Bras Nutr Clin. 2006;21(2): 117-24.

11. Marques APO, Arruda IKG, Leal MCC, Espírito Santos ACG. Envelhecimento, obesidade e consumo alimentar em idosos. Rev Bras Geriatr Gerontol. 2007;10(2):1-9.

12. Brasil. Ministério da Saúde. Vigilância das DANT no contexto da vigilância em saúde no Brasil. Seminário Nacional de Vigilância em Doenças e Agravos Não Transmissíveis e Promoção da Saúde [Internet]. Brasília; 2006 [capturado 2018 out. 02]. 102 p. Disponível em: http://bvsms.saude.gov.br/bvs/publicacoes/anais_doencas agravos.pdf

13. Lazarou C, Matalas AL. Breakfast intake is associated with nutritional status, mediterranean diet adherence, serum iron and fasting glucose: the CYFamilies study. Public Health Nutr. 2014;18(7): 1308-16. https://doi.org/10.1017/S1368980014001967

14. Acu-a K, Cruz T. Avaliação do estado nutricional de adultos e idosos e situação nutricional da população brasileira. Arq Bras Endocrinol Metab. 2004;48(3):345-61. https://doi.org/10.1590/S000427302004000300004

15. Brasil. Vigilância Alimentar e Nutricional. Sisvan: orientações básicas para a coleta, processamento, análise de dados e informação em serviços de saúde. Brasília: Ministério da Saúde; 2011.

16. World Health Organization. Preventing and managing the global epidemic [Internet]. Report of a WHO Consultation. 2000 [capturado 2018 Out 02]. 252 p. Disponível em: http://www.who. int/nutrition/publications/obesity/WHO_TRS_894/en/

17. Lebrão $M L$, Duarte Y. O projeto SABE no município de São Paulo: uma abordagem inicial [Internet]. Brasília: Organização Panamericana da Saúde; 2003 [capturado 2018 Out 02]. 259 p. Disponível em: http://livros01.livrosgratis.com.br/op000002.pdf

18. Associação Brasileira para o Estudo da Obesidade e Síndrome Metabólica. Diretrizes brasileiras de obesidade 2009-2010 [Internet]. Abeso. São Paulo: AC Farmacêutica; 2009 [capturado 2018 Out 02]. 85 p. Disponível em: http://www.abeso.org.br/pdf/ diretrizes_brasileiras_obesidade_2009_2010_1.pdf

19. Pinheiro AB, Lacerda EMA, Benzecry EH, Gomes MCS, Costa VM. Tabela para avaliação de consumo alimentar em medidas caseiras. Rio de Janeiro: Ateneu; 2004.

20. Institute of Medicine. Dietary reference intakes for energy, carbohydrate, fiber fat, fatty acids, cholesterol, protein, and amino acids. Washington: The National Academies Press; 2010.

21. Callegar-Jacqes SM. Bioestatística: princípios e aplicações. Porto Alegre: Artmed; 2003.

22. Cervato AM, Derntl AM, Latorre MRDO, Marcucci MFN. Educação nutricional para adultos e idosos: uma experiência positiva em universidade aberta para a terceira idade. Rev Nutr. 2005;18(1): 41-52. https://doi.org/10.1590/S1415-52732005000100004

23. Instituto Brasileiro de Geografia e Estatística. Síntese de indicadores sociais: uma análise das condições de vida da população brasileira [Internet]. IBGE. Brasília. 2013. [capturado 2016 Mar 24]. 266 p. Disponível em: http://biblioteca.ibge.gov.br/visualizacao/livros/ liv66777.pdf

24. Corteletti IA, Casara MB. Projeto pedagógico: Universidade da Terceira Idade UNTI. Caxias do Sul: Educs; 2007. 
25. Kümpel DA, Sodré AC, Pomatti DM, Scortegagna HM, Filippi J, Portella MR, Doring M, Scariot M. Obesidade em idosos acompanhados pela estratégia de saúde da família. Texto Contexto - Enferm. 2011;20(3):271-7. https://doi.org/10.1590/S010407072011000300007

26. Gallon CW, Wender COM. Estado nutricional e qualidade de vida da mulher climatérica. Ver Bras Ginecol Obstet. 2012;34(4):175-83. https://doi.org/10.1590/S0100-72032012000400007

27. Gonçalves JTT, Silveira MF, Campos MCC, Costa LHR. Sobrepeso e obesidade e fatores associados ao climatério. Cienc Saude Colet. 2016;21(4):1145-55. https://doi.org/10.1590/141381232015214.16552015

28. Catão MHCV, Xavier FC, Pinto TCA. O impacto das alterações do sistema estomatognático na nutrição do idoso. Rev Bras Ciênc Saúde. 2011;9(29):73-8.

29. De Lorenzi DRS, Basso E, Fagundes PO, Saciloto B. Prevalencia de sobrepeso e obesidade no climatério. Bras Ginecol Obstet. 2005;27(8):479-84. https://doi.org/10.1590/S0100-72032005 000800008

30. Galvão NI, Vilela RFJTJ, Orlandi BMM, Ferraz RF, Costa FAA, Fagundes DJ. Determinação do risco cardiovascular em população de check-up espontâneo através do Escore de Framingham. Rev Bras Cardiol. 2013;26(5):356-63.

31. Roman R, Siviero J. Doenças crônicas não transmissíveis e os fatores de risco em mulheres de Guaporé (RS). Ciênc Saúde (Porto Alegre). 2018;11(1):25-32. https://doi.org/10.15448/1983652X.2018.1.25909

32. Linhares RS, Horta BL, Gigante DP, Costa JSD, Olinto MTA. Distribuição de obesidade geral e abdominal em adultos de uma cidade no Sul do Brasil. Cad Saúde Pública. 2012;28(3):438-48. https://doi.org/10.1590/S0102-311X2012000300004

33. Rezende FAC, Rosado LEFPL, Ribeiro RCL, Vidigal FC, Vasques ACJ, Bonard IS, Carvalho CR. Índice de massa corporal e circunferência abdominal: associação com fatores de risco cardiovascular. Arq Bras Cardiol. 2006;87(6):728-34. https://doi.org/10.1590/S0066$782 \times 2006001900008$

34. Nascimento CM, Ribeiro AQ, Sant'Ana LFR, Oliveira RMS, Franceschino SCC, Priore SE. Estado nutricional e condições de saúde da população idosa brasileira: revisão da literatura. Rev Med Minas Gerais. 2011;21(2):174-80.

35. Menezes TN, Marucci MFN. Valor energético total e contribuição percentual de calorias por macronutrientes da alimentação de idosos domiciliados em Fortaleza - CE. Rev Assoc Med Bras. 2012;58(1):3340. https://doi.org/10.1590/S0104-42302012000100012

36. Malta MB, Papini SJ, Corrente JE. Avaliação da alimentação de idosos de município paulista: aplicação do Índice de Alimentação Saudável. Cienc Saude Colet. 2013; 18(2):377-84. https://doi. org/10.1590/S1413-81232013000200009

37. Morimoto JM, Marchioni DML, Cesar CLG, Fisberg RM. Variância intrapessoal para ajuste da distribuição de nutrientes em estudos epidemiológicos. Rev Saúde Públ. 2011;45(3):621-5. https://doi. org/10.1590/S0034-89102011000300022

38. Avelino GF, Previdelli NA, Castro MA, Marchioni DML, Fisberg RM. Sub-relato da ingestão energética e fatores associados em estudo de base populacional. Cad Saúde Pública. 2014;30(3):663-8. https:// doi.org/10.1590/0102-311X00073713
39. Martinazzo J, Zemolin GP, Spinelli RB, Zanardo VPS, Ceni GC. Avaliação nutricional de mulheres no climatério atendidas em ambulatório de nutrição no norte do Rio Grande do Sul, Brasil. Cienc Saúde Colet. 2013;18(11):3349-56. https://doi.org/10.1590/ S1413-81232013001100024

40. Venturini CD, Engroff P, Sgnaolin V, El Kik RM, Morrone FB, Silva Filho IG, De Carlo GA Consumo de nutrientes em idosos residentes em Porto Alegre (RS), Brasil: um estudo de base populacional. Cienc Saude Colet. 2015;20(12):3701-11. https://doi.org/10.1590/1413812320152012.01432015

41. Morley JE, Baumgartner RN, Roubenoff R, Mayer J, Nair KS. Sarcopenia. J Lab Clin Med. 2001;137(4):231-43. https://doi. org/10.1067/mlc.2001.113504

42. Houston DK. Dietary protein intake is associated with lean mass change in older, community-dwelling adults: Health ABC. Am J Clin Nutr. 2008;87(1):150-5. https://doi.org/10.1093/ajcn/87. 1.150

43. Silva WJM, Ferrari CKB. Metabolismo mitocondrial, radicais livres e envelhecimento. Rev Bras. Geriatr Gerontol. 2011;14(3): $441-51$.

44. Araújo MC, Bezerra IN, Barbosa FS, Junger WL, Yokoo EN, Pereira RA, Sichieri R. Consumo de macronutrientes e ingestão inadequada de micronutrientes em adultos. Rev Saúde Públ. 2013;47(1): 177-89. https://doi.org/10.1590/S0034-89102013000700004

45. Coussirat $C$, Batista C, Schneider RH, Resende TL, Schwanke CHA. Vitaminas B12, B6, B9 e homocisteína e sua relação com a massa óssea em idosos. Rev Bras Geriatr Gerontol. 2012;15(3):577-85. https://doi.org/10.1590/S1809-98232012000300018

46. Wong CW. Vitamin B12 deficiency in the elderly: is it worth screening? Hong Kong Med J. 2015;21(2):155-64. https://doi. org/10.12809/hkmj144383

47. Ross AC, Taylor CL, Yaktine AL, Del Valle HB. Dietary reference intakes for calcium and vitamin D [Internet]. Institute of Medicine Committee to Review Dietary Reference Intakes for Vitamin D and Calcium. 2011 [capturado 2018 Out 02]. Disponível em: http:// www.ncbi.nlm.nih.gov/books/NBK56070/

48. Leão ALM, Santos LC. Consumo de micronutrientes e excesso de peso: existe relação? Rev Bras Epidemiol. 2012;15(1):85-95. https:// doi.org/10.1590/S1415-790X2012000100008

49. Chowdhury EA, Richardson JD, Holman GD, Tsintzas K, Thompson D, Betts JA. The causal role of breakfast in energy balance and health: a randomized controlled trial in obese adults. Am J Clin Nutr. 2016;(103):747-56. https://doi.org/10.3945/ajcn.115. 122044

50. Freitas PP, Mendonça RD, Lopes ACS. Factors associated with breakfasting in users of a public health service. Rev Nutr. 2013;26(2):195-203. https://doi.org/10.1590/S141552732013000200007

51. Trancoso SC, Cavalli SB, Proença RPC. Café da manhã: caracterização, consumo e importância para a saúde. Rev Nutr. 2010;23(5):859-69. https://doi.org/10.1590/S1415-52732010000500016

52. Jakubowicz D, Barnea M, Wainstein J, Froy O. High caloric intake at breakfast vs. dinner differentially influences weight loss of overweight and obese women. Obesity. 2013;21(12):2504-12. https://doi.org/10.1002/oby.20460 
53. Molz P, Pereira CS, Gassen TL, Prá D, Franke SIR. Relação do consumo alimentar de fibras e da carga glicêmica sobre marcadores glicêmicos, antropométricos e dietéticos em pacientes pré-diabéticos. Rev Epidemiol Controle Infecç. 2015;5(3):131-5. https://doi.org/10.17058/reci.v5i3.5585

54. Bernaud FSR, Ticiana CR. Fibra alimentar: ingestão adequada e efeitos sobre a saúde do metabolismo. Arq Bras Endocrinol Metab. 2013;57(6):397-405. https://doi.org/10.1590/S000427302013000600001

55. Barazzetti R, Siviero J, Bonatto S. Estado nutricional, consumo de calorias e macronutrientes de mulheres participantes de uma universidade da terceira idade no sul do país. Estud Interdiscipl Envelhec. 2011;18(2):331-47.
56. Rodrigues APS, Silveira EA. Correlação e associação de renda e escolaridade com condições de saúde e nutrição em obesos. Cienc Saude Colet. 2015;20(1):165-74. https://doi.org/10.1590/141381232014201.18982013

57. Bains K, Kaur G, Kaur H, Kaur A. Assessment of changes in anthropometry and body composition with progression of age among occupationally sedentary adult women. Int J Health Nutr. 2012;3(1):13-8.

58. Guimarães ECM, Santos LS, Jesus BM, Pastana, NA. Sarom MLG. Perfil nutricional de idosas frequentadoras da Faculdade da Terceira Idade. Cad UniFOA. 2009;4(10):67-72. 